\title{
Nanocrystal-Loaded Micelles for the Enhanced In Vivo Circulation of Docetaxel
}

\author{
Meng Cheng ${ }^{1,+}$, Qiaoming Liu ${ }^{1,2,+}$, Tiantian Gan ${ }^{1}$, Yuanying Fang ${ }^{1}$, Pengfei Yue ${ }^{1}$, Yongbing Sun ${ }^{1}$, Yi Jin ${ }^{1}$, \\ Jianfang Feng $1,2, *$ and Liangxing Tu $1, *(\mathbb{B})$
}

1 National Pharmaceutical Engineering Center for Solid Preparation in Chinese Herbal Medicine, Jiangxi University of Traditional Chinese Medicine, Nanchang 330006, China; cmjxzyy@126.com (M.C.); liuqm.to12@foxmail.com (Q.L.); gantt_1@126.com (T.G.); fangyuanying@163.com (Y.F.); ypfpharm@126.com (P.Y.); yongbing_sun@hotmail.com (Y.S.); jinyizju@hotmail.com (Y.J.)

2 School of Pharmacy, Guangxi University of Chinese Medicine, Nanning 530200, China

* Correspondence: fengjianfang@vip.163.com (J.F.); tufrankie@163.com (L.T.); Tel.: +86-188-1733-8957 (L.T.)

+ These authors contributed equally to this work.

Citation: Cheng, M.; Liu, Q.; Gan, T.; Fang, Y.; Yue, P.; Sun, Y.; Jin, Y.; Feng, J.; Tu, L. Nanocrystal-Loaded Micelles for the Enhanced In Vivo Circulation of Docetaxel. Molecules 2021, 26, 4481 . https://doi.org/10.3390/

molecules 26154481

Academic Editors: Yi Lu and

Yongtai Zhang

Received: 21 June 2021

Accepted: 18 July 2021

Published: 24 July 2021

Publisher's Note: MDPI stays neutral with regard to jurisdictional claims in published maps and institutional affiliations.

Copyright: (c) 2021 by the authors. Licensee MDPI, Basel, Switzerland. This article is an open access article distributed under the terms and conditions of the Creative Commons Attribution (CC BY) license (https:// creativecommons.org/licenses/by/ $4.0 /)$.

\begin{abstract}
Prolonging in vivo circulation has proved to be an efficient route for enhancing the therapeutic effect of rapidly metabolized drugs. In this study, we aimed to construct a nanocrystal-loaded micelles delivery system to enhance the blood circulation of docetaxel (DOC). We employed highpressure homogenization to prepare docetaxel nanocrystals (DOC $(\mathrm{Nc})$ ), and then produced docetaxel nanocrystal-loaded micelles (DOC(Nc)@mPEG-PLA) by a thin-film hydration method. The particle sizes of optimized DOC(Nc), docetaxel micelles (DOC@mPEG-PLA), and DOC(Nc)@mPEG-PLA were $168.4,36.3$, and $72.5 \mathrm{~nm}$, respectively. The crystallinity of docetaxel was decreased after transforming it into nanocrystals, and the crystalline state of docetaxel in micelles was amorphous. The constructed DOC(Nc)@mPEG-PLA showed good stability as its particle size showed no significant change in 7 days. Despite their rapid dissolution, docetaxel nanocrystals exhibited higher bioavailability. The micelles prolonged the retention time of docetaxel in the circulation system of rats, and DOC(Nc)@mPEG-PLA exhibited the highest retention time and bioavailability. These results reveal that constructing nanocrystal-loaded micelles may be a promising way to enhance the in vivo circulation and bioavailability of rapidly metabolized drugs such as docetaxel.
\end{abstract}

Keywords: nanocrystals; micelles; circulation; in vivo; docetaxel

\section{Introduction}

Docetaxel (DOC), a typical taxane, has been approved as a first-line anti-tumor drug in clinical treatments [1,2]. Like other taxanes (paclitaxel, cabazitaxel, etc. [3]), docetaxel primarily operates its anti-tumor efficacy by disrupting the microtubular network, inducing a sustained block at the metaphase-anaphase boundary during cell division [4]. As a compound belonging to BCS IV, the only commercially available formulation containing docetaxel is intravenous injection (such as Taxotere ${ }^{\circledR}$ and Docetaxe ${ }^{\circledR}$ ), in which polysorbate 80 and dehydrated alcohol are used as cosolvents. Unfortunately, these cosolvents contribute to the majority of side reactions, including hypersensitivity, febrile neutropenia, fatigue, fluid retention, and peripheral neuropathy, that docetaxel injections produce, hence limiting its clinical application. Oral administration is the safest and most convenient administration route. Over the past decades, researchers have launched numerous strategies, such as nanoparticles [5,6], nano-emulsion [7], proniosomes [8], liposomes [9], and prodrugs [10], to enhance the oral bioavailability of docetaxel; however, low bioavailability still hinders the development of oral formulations for docetaxel. In this case, developing intravenous injections with higher safety and efficacy is an additional and promising strategy. Considering docetaxel is a rapidly metabolized drug, enhancing its in vivo circulation may benefit its therapeutic efficacy. 
Micelles are self-assembled core-shell nanocarriers formed by surfactants or polymers. Considering their advantages, such as their ease of production, high drug loading (up to 30\%), small particle sizes (below $200 \mathrm{~nm}$ ), and ease of modification [11-13], micelles are a good choice for enhancing the safety and therapeutic efficacy of intravenous formulations [14]. Due to misunderstandings about the mechanism of micellar solubilization, micelles are sometimes regarded as the "solution" of the solutes, but this is not the case [15]. Hence, micelles can exhibit different pharmacokinetic performance to those of intravenous injection solutions. In general, due to their hydrophilic shell, micelles can signal the recognition of the reticuloendothelial system (RES), and thereby decrease the clearance ratio of the RES, resulting in a prolonged in vivo circulation [16,17]. To further enhance the in vivo circulation of micelles, researchers have employed various materials to modify the surface of micelles; one typical responsive is PEGylation [18,19]. With the aid of polyethylene glycol (PEG) and its derivatives, the hydrophilic profile of the micelle surface is improved [20], thus enhancing their blood circulation. However, in recent years, polymeric micelles have increasingly been formed by polymers synthesized from more than three different materials, with the increasing complexity of polymers raising concerns regarding the safety of materials and the commercial viability of these polymer micelles. These concerns have limited the application of the classical strategy (surface modification with materials) for enhancing in vivo circulation of micelles. Therefore, we hypothesized that combining micelles with other formulations that may improve blood circulation, may be a promising way to further enhance the in vivo circulation of micelles.

Nanocrystals, also called nanosuspension, is an additional technology for developing intravenous injection. Owing to its advantages such as high drug loading (up to 100\%), ease of scale-up, and its organic, solvent-free preparation process (top-down method [21]), nanocrystals technology has exhibited great potential in numerous delivery systems. To date, there are over 10 products based on nanocrystals that have been approved by the FDA for oral administration (like Rapamune ${ }^{\circledR}$, Emend $^{\circledR}$ ), and intramuscular injection (such as Invega Sustenna ${ }^{\circledR}$ and Aristada $\left.{ }^{\circledR}\right)$ [22-25]. Though some researchers have reported that some nanocrystals may not alter or improve the pharmacokinetic performance of drugs delivered via intravenous administration [26-28], a more common observation has been that nanocrystals may enhance the retention time and bioavailability of coarse drugs. As a foreign substance, nanocrystals are expected to be uptaken by the mononuclear phagocyte system (MPS) [29]; however, the internalization of nanocrystals in macrophages is limited (about $68 \%$ of ingested nanocrystals remained intact in macrophages $24 \mathrm{~h}$ post uptake $[30,31])$. The undegraded nanocrystals can be released intact with a similar size and shape to the original nanocrystals, hence achieving a prolonged in vivo circulation [32-34]. However, what should be borne in mind is that, as a pure drug system, few modifications can be made to nanocrystals, limiting the potential to further improve circulation time using functional materials. Embedding nanocrystals in another easily modified delivery system may improve their in vivo circulation.

Considering the statements above, we aimed to construct a novel nanocrystal-loaded micelles delivery system to improve the in vivo circulation of docetaxel, docetaxel nanocrystals, and docetaxel micelles. Herein, we prepared docetaxel nanocrystals (DOC(Nc) by using high-pressure homogenization, and then embedded DOC $(\mathrm{Nc})$ into the inner core of the micelles by using mPEG-PLA as their structural material. Figure 1 illustrates the preparation scheme of docetaxel nanocrystal-loaded micelles (DOC(Nc)@mPEG-PLA). 


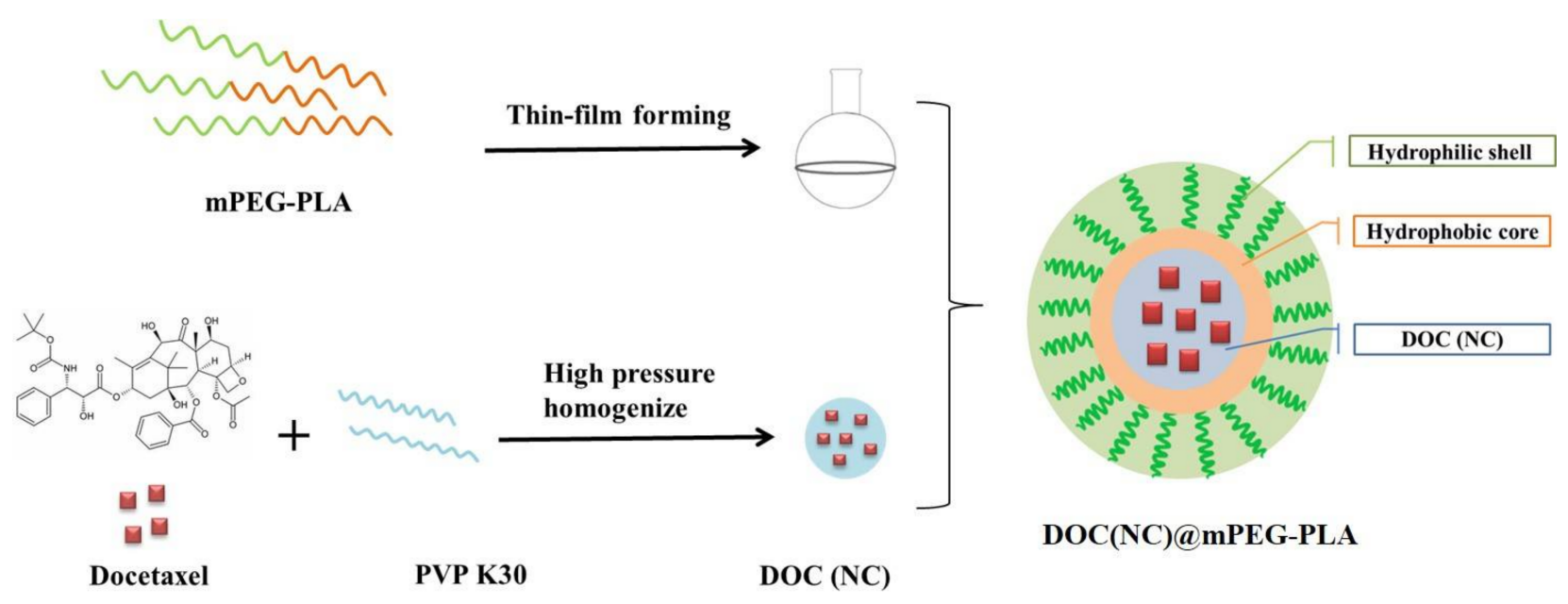

Figure 1. The preparation scheme of docetaxel nanocrystal-loaded micelles (DOC(Nc)@mPEG-PLA).

\section{Results and Discussion}

\subsection{Preparation of DOC(Nc)@mPEG-PLA}

To prepare fine docetaxel nanocrystal-loaded micelles (DOC(Nc)@mPEG-PLA) with small particle sizes, high encapsulation efficacy (EE), and drug loading (DL), we optimized the preparation parameters via mPEG-PLA usage and hydration temperatures, and the results are shown in Figure 2. Though drug loading was increased and mPEG-PLA usage was decreased (Figure 2B), the smallest particle size with high encapsulation efficacy was gained when the mPEG-PLA usage was $150 \mathrm{mg}$ (Figure 2A); hence, we selected $150 \mathrm{mg}$ as the usage of mPEG-PLA. Hydration temperature was another essential factor affecting the construction of micelles, and in this study, we set the hydration temperature at 4, 25, 37 , and $45{ }^{\circ} \mathrm{C}$. We observed that the particle sizes of DOC(Nc)@mPEG-PLA increased as hydration temperature increased, while the highest DL and EE were reached at $25^{\circ} \mathrm{C}$. Therefore, we selected $25^{\circ} \mathrm{C}$ as the hydration temperature.

Combining the results shown above, we achieved the optimized preparation process of DOC(Nc)@mPEG-PLA, which can be described as follows: $150 \mathrm{mg}$ mPEG-PLA is dispersed in $10 \mathrm{~mL} 95 \%$ ethanol, before a thin film is formed by removing the ethanol under $50{ }^{\circ} \mathrm{C}$.

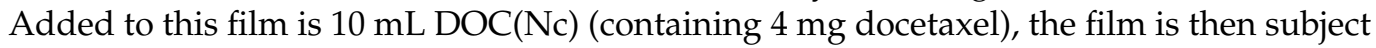
to sonication for $3 \mathrm{~min}$, holding for $30 \mathrm{~min}$ at $25^{\circ} \mathrm{C}$.

\subsection{Characterization of $D O C(N c) @ m P E G-P L A$}

As shown in Table 1, The particle sizes of DOC(Nc) were $168.4 \mathrm{~nm}$. After embedding docetaxel nanocrystals into the inner hydrophobic core of micelles, the particles sizes of docetaxel micelles (DOC@mPEG-PLA) were slightly increased from $36.3 \mathrm{~nm}$ to $72.5 \mathrm{~nm}$. The DL and EE of DOC@mPEG-PLA were $4.89 \%$ and $76.75 \%$, respectively, and those of DOC(Nc)@mPEG-PLA were 1.05\% and 33.51\%, respectively. The decreased DL and EE of nanocrystal-loaded micelles may be explained by the way nanocrystals may enhance the hydrophilicity of docetaxel, thus interrupting the entry process of nanocrystals into the inner hydrophobic core of micelles. 

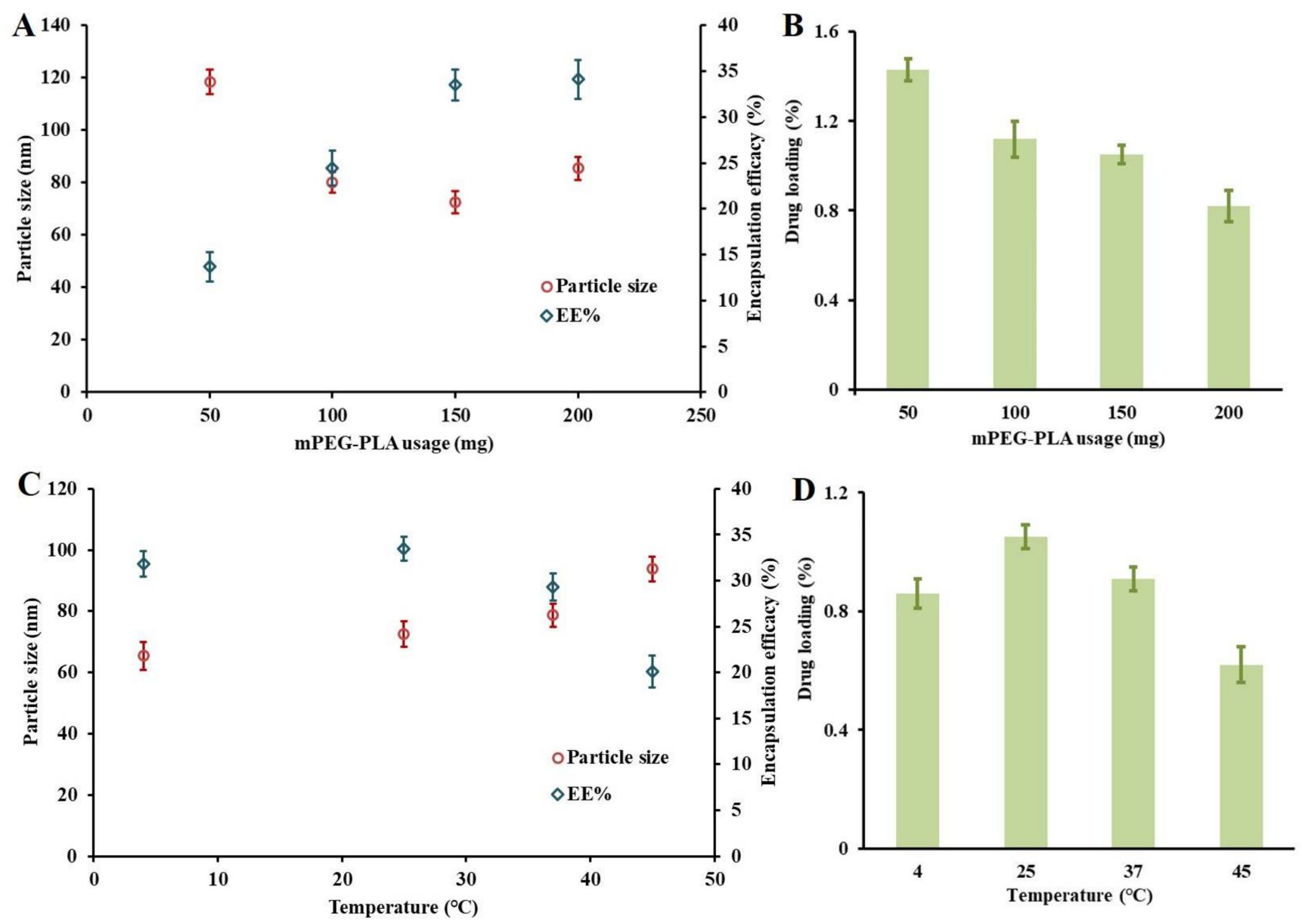

Figure 2. The effects of mPEG-PLA usage and hydration temperatures on the particle sizes, or encapsulation efficacy (A,C), and drug loading (B,D) of DOC(Nc)@mPEG-PLA. $(n=3)$.

Table 1. The particle sizes, drug loading (DL), and encapsulation efficiency (EE) of different formulations. (mean $\pm \mathrm{SD}, n=3$ ).

\begin{tabular}{cccc}
\hline & Sizes $(\mathbf{n m})$ & DL (\%) & EE (\%) \\
\hline DOC(Nc) & $168.4 \pm 11.9$ & N/A & N/A \\
DOC@mPEG-PLA & $36.3 \pm 2.0$ & $4.89 \pm 0.07$ & $76.75 \pm 0.62$ \\
DOC(Nc)@mPEG-PLA & $72.5 \pm 4.2$ & $1.05 \pm 0.04$ & $33.51 \pm 1.70$ \\
\hline
\end{tabular}

As shown in Figure 3A, the crude docetaxel had an irregular shape and its diameter was on the micron scale. After transferring the docetaxel into nanocrystals (DOC $(\mathrm{Nc})$ ) or micelles (DOC@mPEG-PLA), the morphology became more spherical (Figure 3B,C). Because of the embedding of nanocrystals, the diameter of micelles slightly increased, and the nanocrystal-loaded micelles (DOC $(\mathrm{Nc}) @ \mathrm{mPEG}-\mathrm{PLA})$ became more spherical (Figure 3D). The particle sizes of DOC $(\mathrm{Nc})$, DOC $\left(\mathrm{Nc}_{\mathrm{c}} @ \mathrm{mPEG}-\mathrm{PLA}\right.$, and DOC $(\mathrm{Nc}) @ \mathrm{mPEG}-\mathrm{PLA}$ observed in TEM spectra were similar to that detected by Nano ZS 90 nanoparticle size and zeta potential meter (Malvern Instruments Co. Ltd., Malvern, UK). 

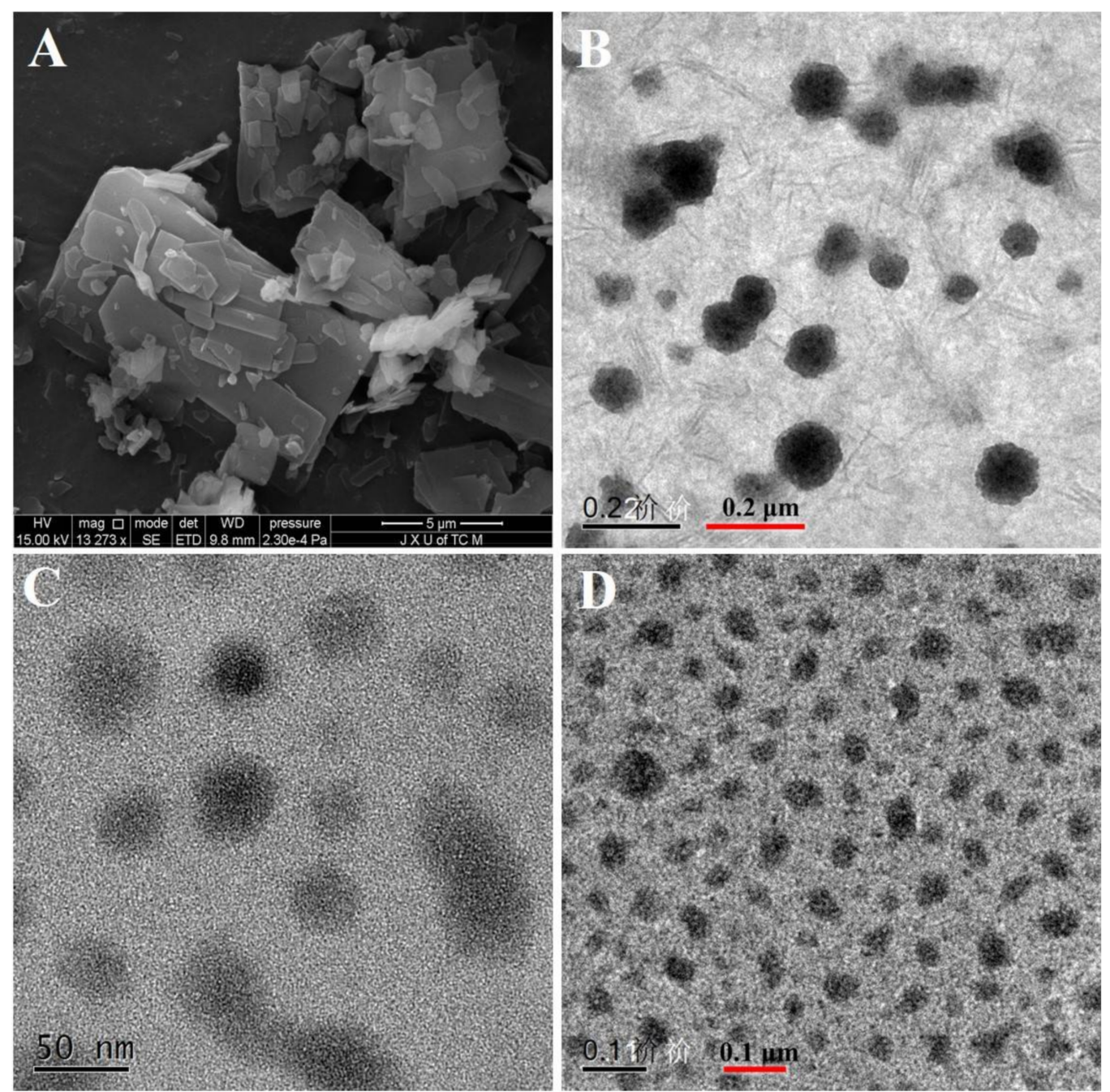

Figure 3. The SEM photo of DOC (A); the TEM photos of DOC(Nc) (B), DOC@mPEG-PLA (C), and DOC(Nc)@mPEG-PLA (D).

In this study, we employed x-ray diffraction (XRD) (Ultima IV, Rigaku, Tokyo, Japan) and differential scanning calorimetry (DSC) (DSC Q2000, TA Instruments, New Castle, DE, America) to explore the crystal transition of docetaxel during the preparation processes of nanocrystals, micelles, and nanocrystal-loaded micelles. As shown in Figure 4, the coarse docetaxel is in a crystalline state, with its crystallinity drastically decreased in the production process of nanocrystals as there are only several weak diffraction peaks in the XRD spectra of DOC $(\mathrm{Nc})$. Few diffraction peaks of docetaxel were left when transferring docetaxel to docetaxel micelles, revealing that DOC@mPEG-PLA was almost in an amorphous state. Excluding the influence of diffraction peaks of excipients (PVP K30 and mPEG-PLA), there are no obvious diffraction peaks of docetaxel in the XRD spectra of DOC(Nc)@mPEG-PLA, indicating the nanocrystal-loaded micelles were in amorphous state. 


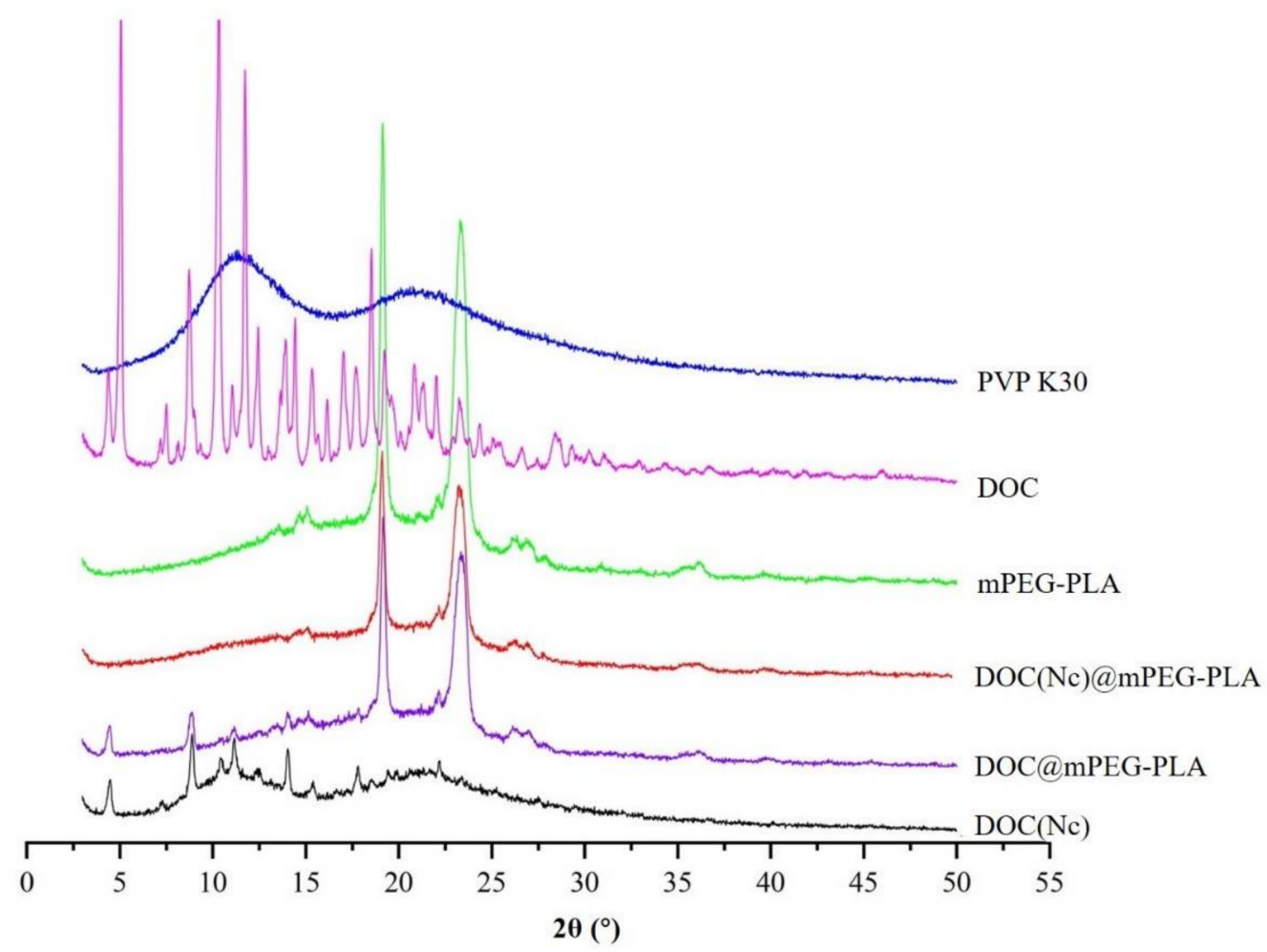

Figure 4. The XRD spectra of different materials.

The DSC spectra were drawn at scanning temperatures ranging from 30 to $300{ }^{\circ} \mathrm{C}$ with a scan rate of $10^{\circ} \mathrm{C} / \mathrm{min}$. In the DSC spectra of docetaxel, endothermic peaks at about $220^{\circ} \mathrm{C}$ can be observed; however, after treatments of preparation processes of nanocrystals, micelles, and nanocrystal-loaded micelles, these endothermic peaks are absent (Figure 5). Combining the results of the XRD and DSC spectra, it is certain that the docetaxel had undergone crystal transition in the preparation processes of the different formulations.

\subsection{Release Profile of $D O C(N c) @ m P E G-P L A$}

As in vitro release profile typically has good correlation to in vivo performance, we operated a release study to better understand the in vivo pharmacokinetic behaviors of different formulations. The in vitro release behaviors of DOC, DOC $(\mathrm{Nc})$, DOC@mPEGPLA, and DOC(Nc)@mPEG-PLA were studied by dialysis performed in a PBS buffer ( $\mathrm{pH}$ 7.4), which could be used to simulate the conditions in blood circulation. Owing to its decreased particle sizes and diffusion layer, $\mathrm{DOC}(\mathrm{Nc})$ released faster than DOC [35], and the release rate of DOC slowed down after transferring it into micelles, which may be attributed to factors such as hydrophobicity of the inner core of micelles and hydrogen bonding between molecules [36-38]. The release rate of DOC(Nc)@mPEG-PLA was faster than DOC@mPEG-PLA, and slower than DOC (Figure 6). 


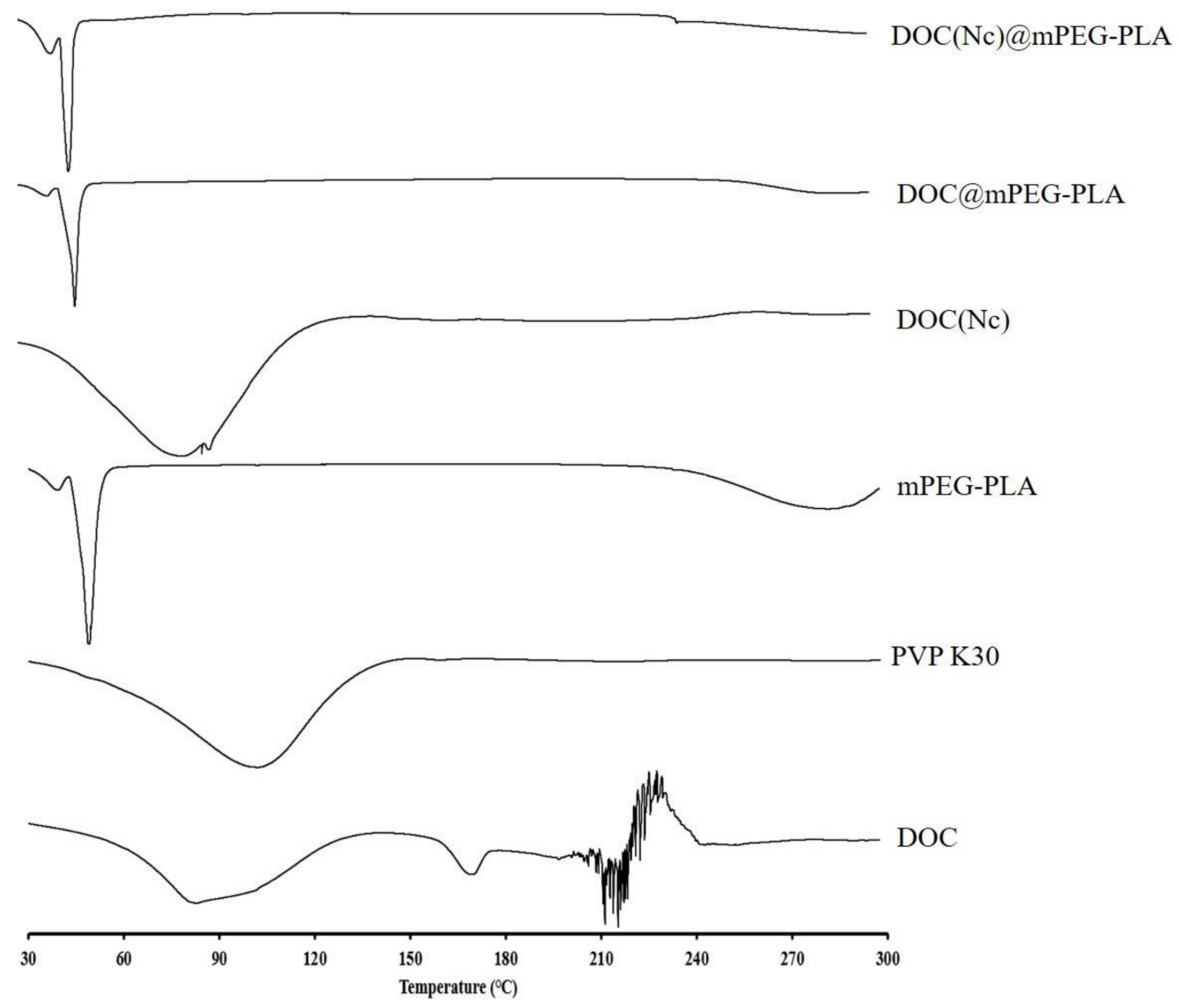

Figure 5. The DSC spectra of different materials.

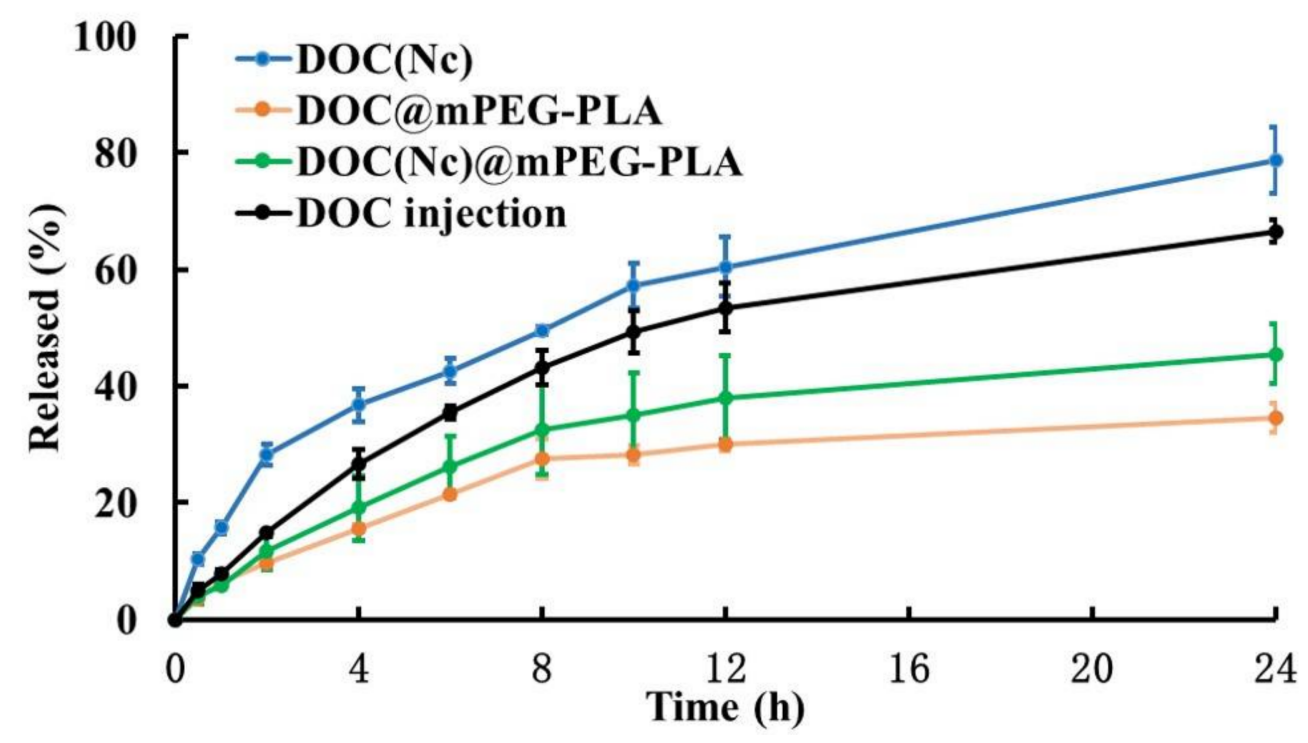

Figure 6. Release profiles of different formulations in a PBS buffer $(\mathrm{pH} 7.4)$ at $37^{\circ} \mathrm{C}(n=3)$.

\subsection{Stability of DOC(Nc)@mPEG-PLA}

After placing the DOC $(\mathrm{Nc})$, DOC@mPEG-PLA, and DOC(Nc)@mPEG-PLA at room temperature for 7 days, the particle sizes of each sample at $0,1,2,3$, and 7 days were detected. Figure 7 illustrates that there was no significant change to particle sizes during the experimental period, revealing that these three formulations had good stability at room 
temperature without any dilution. In addition, after incubating with a PBS buffer or PBS buffer containing $10 \%$ FBS (10\% FBS-PBS buffer) for $8 \mathrm{~h}$, the particle sizes of DOC(Nc), DOC@mPEG-PLA, and DOC(Nc)@mPEG-PLA were changed from $173.5 \pm 8.3,33.8 \pm 1.9$, and $77.9 \pm 3.3$ to $176.4 \pm 5.3,30.7 \pm 2.8$, and $75.2 \pm 4.9$, respectively, in PBS buffer, while in $10 \%(v / v)$ FBS-PBS buffer, the particle sizes were changed from $191.4 \pm 15.3,42.8 \pm 1.7$, and $83.5 \pm 5.0$ to $382.5 \pm 21.4,57.2 \pm 3.8$, and $101.3 \pm 6.7$ for DOC(Nc), DOC@mPEG-PLA, and DOC(Nc)@mPEG-PLA, respectively. These results show that these nano formulations were stable in a PBS buffer; however, after incubating with serum, the particle sizes of nanocrystals largely increased, which may be due to the drug-protein interaction [39], and, due to the excellent hydrophilic property of $\mathrm{MPEG}$, the micelles and nanocrystal-loaded micelles exhibited good stability [40].

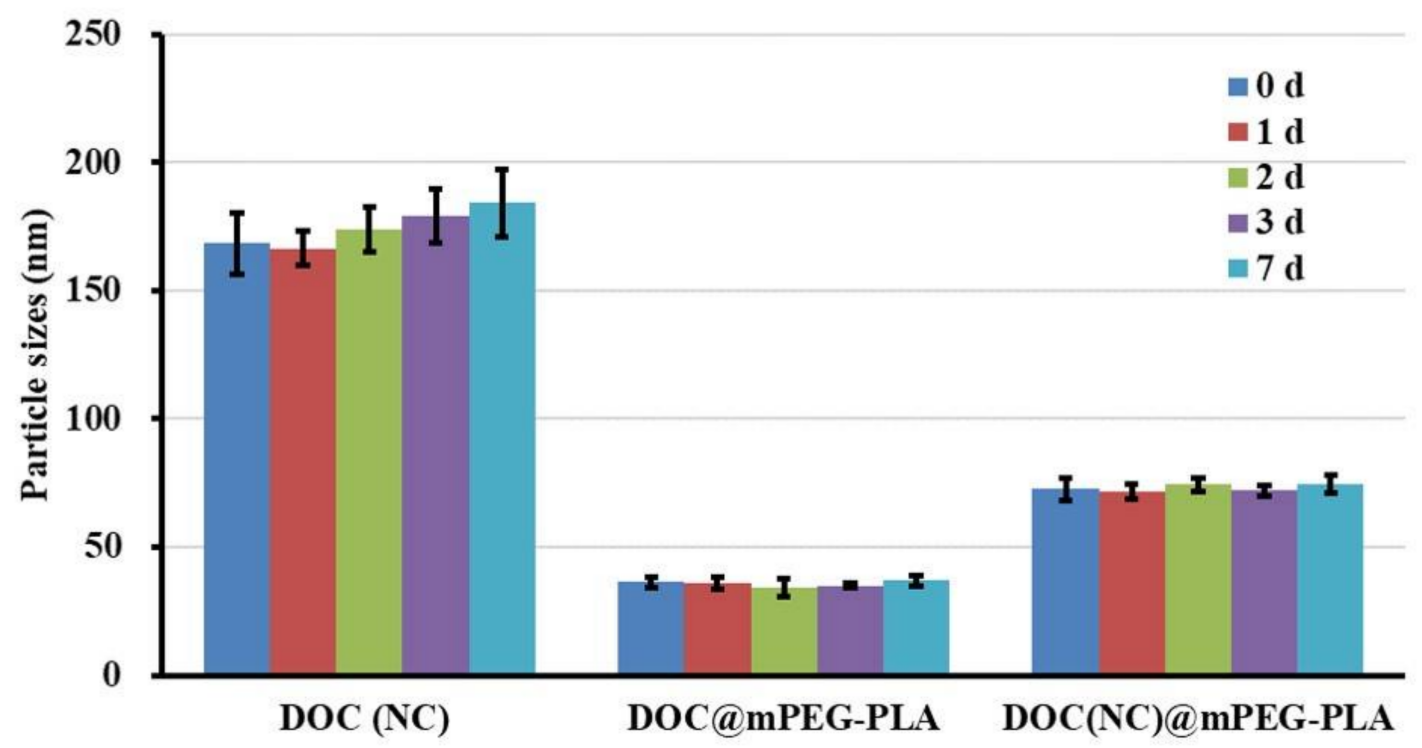

Figure 7. The particle sizes of DOC(Nc), DOC@mPEG-PLA, and DOC(Nc)@mPEG-PLA after holding at room temperature for 7 days. $(n=3)$.

\subsection{Pharmacokinetic Behavior of DOC(Nc)@mPEG-PLA}

It is certain that the pharmacokinetic behavior of DOC changed, as shown in Table 2 and Figure 8. The $\mathrm{C}_{1 \mathrm{~min}}$ of DOC injection (Taxotere ${ }^{\circledR}$ ) was $3947 \mathrm{ng} / \mathrm{mL}$, while that for DOC (NC) was 11,181 ng/mL. In addition, the bioavailability of DOC (NC) was higher than that of DOC injection. The enhanced $\mathrm{C}_{1 \mathrm{~min}}$ and bioavailability of DOC (NC) can be explained by nanocrystals being uptaken by the reticuloendothelial system (RES) [41], preventing the rapid metabolization of DOC by the liver and allowing some nanocrystals to escape from the RES without being internalized [30]. The $\mathrm{C}_{1 \mathrm{~min}}$ of DOC injection and DOC@mPEG-PLA were similar; however the bioavailability of DOC was enhanced by micelles, which may be caused by their sustained release and their capacity to escape the RES [42-44]. DOC(NC)@mPEG-PLA exhibited the highest $\mathrm{C}_{1 \mathrm{~min}}$ and bioavailability, which were 3.58- and 2.69-fold, respectively, to DOC injection. In addition, compared to DOC injection, DOC (NC), and DOC@mPEG-PLA, DOC(NC)@mPEG-PLA showed decreased $C L$ and enhanced MRT and $t_{1 / 2}$. These results reveal the in vivo performance of DOC(NC)@mPEG-PLA: firstly, the docetaxel nanocrystals underwent sustained release from DOC(NC)@mPEG-PLA; subsequently, DOC(NC) was uptaken by the RES; before finally, the DOC(NC) excited the RES by diffusing down the drug concentration gradient. With the enhanced blood circulation of nanocrystals and the sustained-release behavior of micelles, the nanocrystal-micelles system showed the largest potential for enhancing in vivo circulation and bioavailability of rapidly metabolized drugs such as docetaxel. 
Table 2. Pharmacokinetics parameters of different formulations on rats via intravenous route at $10 \mathrm{mg} / \mathrm{kg}(\mathrm{mean} \pm \mathrm{SD}, n=5)$.

\begin{tabular}{cccccc}
\hline Parameters & Units & DOC Injection & DOC (NC) & DOC@mPEG-PLA & DOC(NC)@mPEG-PLA \\
\hline $\mathrm{AUC}(0 \rightarrow \infty)$ & $\mathrm{ng} / \mathrm{L} \mathrm{h}$ & $1313 \pm 342$ & $2542 \pm 405^{*}$ & $2084 \pm 517^{*}$ & $3532 \pm 157^{*, \#,+}$ \\
$\mathrm{MRT}(0 \rightarrow \infty)$ & $\mathrm{h}$ & $7.197 \pm 3.436$ & $5.545 \pm 2.185$ & $10.387 \pm 2.142^{*}$ & $15.668 \pm 2.520^{*}, \#,+$ \\
$\mathrm{t}_{1 / 2}$ & $\mathrm{~h}$ & $7.334 \pm 4.212$ & $7.886 \pm 3.831$ & $9.945 \pm 2.627$ & $17.504 \pm 3.362 *, \#,+$ \\
$\mathrm{CL}$ & $\mathrm{L} / \mathrm{h} / \mathrm{Kg}$ & $0.008 \pm 0.003$ & $0.004 \pm 0.001^{*}$ & $0.005 \pm 0.002$ & $0.003 \pm 0.001^{*,+}$ \\
$\mathrm{C}_{1 \text { min }}$ & $\mathrm{ng} / \mathrm{L}$ & $3947 \pm 914$ & $11,181 \pm 4741^{*}$ & $3892 \pm 1274$ & $14,120 \pm 845^{*}+{ }^{*}$ \\
\hline
\end{tabular}

${ }^{*} p<0.05$ vs. DOC injection, ${ }^{*} p<0.05$ vs. DOC (NC), ${ }^{+} p<0.05$ vs. DOC@mPEG-PLA.

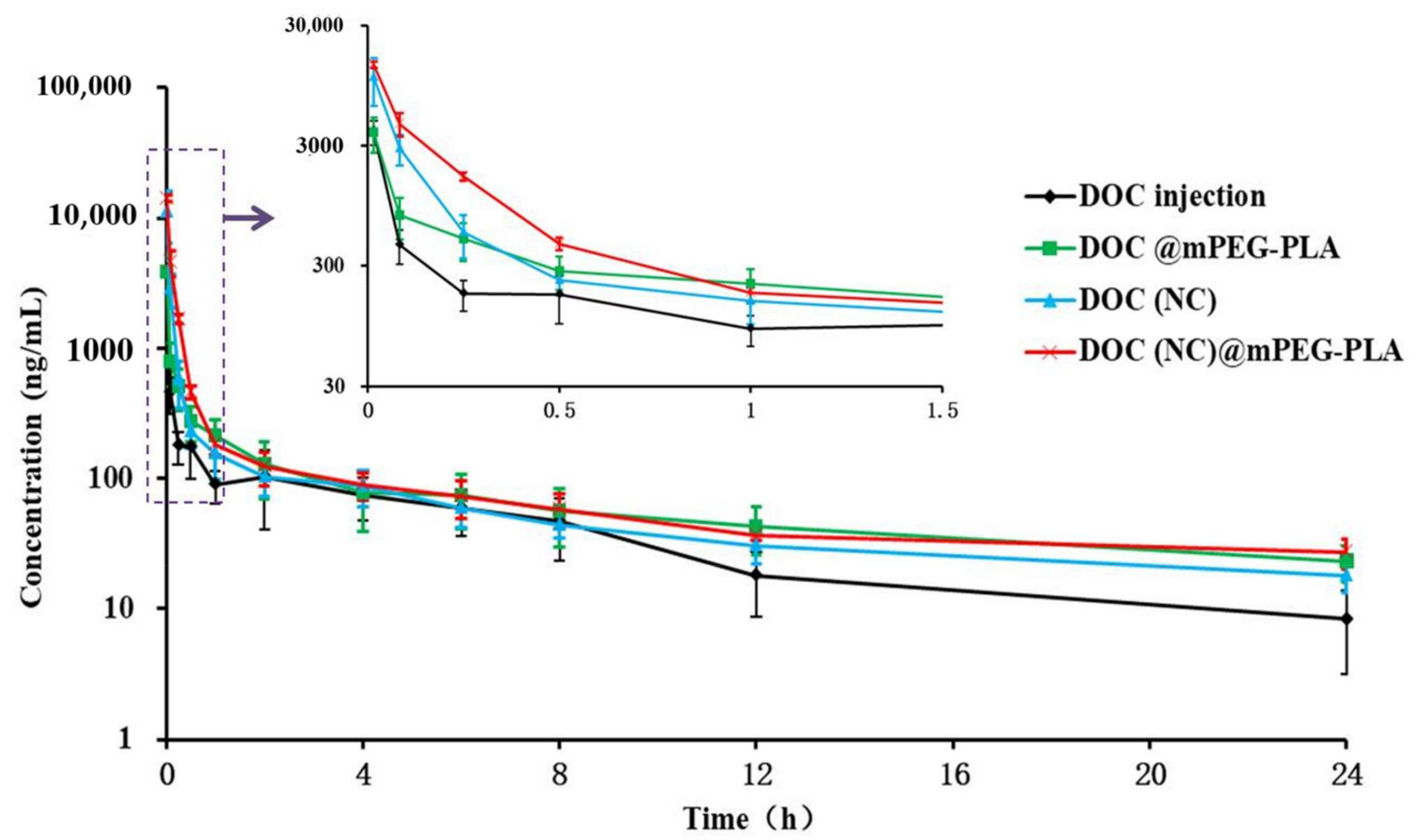

Figure 8. The concentration-time curves of different formulations on rats via intravenous route at $10 \mathrm{mg} / \mathrm{kg}(n=5)$.

Owing to the ease of modifying its surface behavior, micelles, modified with multiple functional agents, may enhance the in vivo performance of docetaxel, such as when it is modified by monomethoxy poly(ethylene glycol)-poly(epsilon-caprolactone) (mPEGPCL) [45] and stearic acid (SA)-modified Bletilla striata polysaccharides (BSPs) copolymers [46]. However, studies have primarily focused on how to enhance targeting efficacy and cell/tissue penetration ability, while attempts to fabricate multi-nanoparticle embedded complexes to enhance the in vivo circulation have been crude. As for nanocrystals, several researchers have attempted to enhance the in vivo performance of docetaxel nanocrystals, for example, after modification with apo-Transferrin human (Tf) [47] or trans-activator of transcription (TAT) peptide [48], the anti-tumor efficacy of unmodified docetaxel micelles was enhanced. However, what needs to be emphasized is that, as a pure drug system, modification of nanocrystals is limited. Hence, with the construction of nanocrystal micelles, we can use the enhanced in vivo circulation ability of nanocrystals to enhance the circulation time of micelles, and can similarly take the advantages of micelles (such as enhanced circulation time and ease of modification) to improve the in vivo performance of nanocrystals. 


\section{Materials and Methods}

\subsection{Materials}

Docetaxel (purity $>99 \%$ ) and paclitaxel (purity $>99 \%$ ) were purchased from Wuhan Zeshancheng Biotechnology Co., Ltd., Wuhan, China. Povidone K30 (PVP K30) was purchased from Boai NKY Pharmaceuticals Ltd., Jiaozuo, China. Methoxy polyethylene glycol-b-poly(L-lactide) (mPEG-PLA) was supplied by Sigma-Aldrich, City of Saint Loui, USA. Phosphate-buffered saline (PBS) was gained from Aladdin, Shanghai, China. Fetal bovine serum (FBS) was gained from Gibco, New York, USA. All other materials and reagents were of analytical grade and purified water was used throughout this study.

Male Sprague-Dawley (SD) rats, weighing $250 \pm 20$ g, were supplied by the Hunan STJ Laboratory Animal Co., Ltd. (Changsha, China).

\subsection{Preparation of Docetaxel Nanocrystals (DOC $(\mathrm{Nc})$ )}

The docetaxel nanocrystals were prepared via high-pressure homogenization technology. We dispersed $20 \mathrm{mg}$ docetaxel in $50 \mathrm{~mL}$ purified water (containing $100 \mathrm{mg}$ PVP K30), and then pre-treated by a high shear homogenizer (Fluko ${ }^{\circledR}$ FA25, FLUKO, Shanghai, China) at 13,000 rpm for $10 \mathrm{~min}$. The crude suspension was treated in a high-pressure homogenizer (AH NANO, ATS, Shanghai, China) thereafter. The docetaxel nanocrystals were harvested after homogenizing at 1000 bar for 20 cycles.

\subsection{Preparation of Docetaxel Micelles (DOC@mPEG-PLA)}

The docetaxel micelles were prepared by using a thin-film hydration method. Briefly, $10 \mathrm{mg}$ docetaxel and $150 \mathrm{mg}$ mPEG-PLA were dispersed in $10 \mathrm{~mL} 95 \%$ ethanol, and the ethanol was removed by evaporation under $50{ }^{\circ} \mathrm{C}$ to form a thin film, which was hydrated by $10 \mathrm{~mL}$ purified water under sonication for $3 \mathrm{~min}$ and holding for $30 \mathrm{~min}$ at $25^{\circ} \mathrm{C}$. The docetaxel micelles were gained after filtering the unencapsulated DOC by using $0.22 \mu \mathrm{m}$ microporous membrane.

\subsection{Preparation of Docetaxel Nanocrystals Loaded Micelles (DOC(Nc)@mPEG-PLA)}

Several mPEG-PLA were dispersed in $10 \mathrm{~mL} 95 \%$ ethanol, and then the thin film was formed by removing the ethanol under $50{ }^{\circ} \mathrm{C} .10 \mathrm{~mL} \mathrm{DOC}(\mathrm{Nc})$, prepared as Section 3.2., was added to the thin film, and underwent sonication for $3 \mathrm{~min}$, holding for $30 \mathrm{~min}$ at pre-set temperatures. The unencapsulated $\mathrm{DOC}(\mathrm{Nc})$ was removed by centrifugation at $12,000 \mathrm{rpm}$ for $10 \mathrm{~min}$, followed by filtration through $0.22 \mu \mathrm{m}$ microporous membrane. The effects of mPEG-PLA usage and hydration temperatures on the particle sizes, DL, and EE of DOC(Nc)@mPEG-PLA were studied.

\subsection{Characterization of DOC(Nc)@mPEG-PLA \\ 3.5.1. Particle Sizes}

The particle sizes of DOC(Nc), DOC@mPEG-PLA, and DOC(Nc)@mPEG-PLA were characterized by Nano ZS 90 nanoparticle size and zeta potential meter (Malvern Instruments Co., Ltd., Malvern, UK), and all samples were analyzed in triplicate.

\subsubsection{Drug Loading and Encapsulation Efficacy}

The drug loading (DL) and encapsulation efficiency (EE) of DOC@mPEG-PLA and DOC(Nc)@mPEG-PLA were measured as reported prior [49,50]. In brief, $2 \mathrm{~mL}$ methanol was added to $100 \mu \mathrm{L}$ DOC@mPEG-PLA or DOC(Nc)@mPEG-PLA by vortexing for $1 \mathrm{~min}$ to destroy the structure of the micelles, and then the samples were centrifugated at $12,000 \mathrm{rpm}$ for $10 \mathrm{~min}$. The drug concentration of the supernatant was detected by HPLC which was performed as follows: employing Ultimate XB-C18 column $(4.6 \times 250 \mathrm{~mm}$, $5 \mu \mathrm{m})$ as the detection column, selecting acetonitrile-water solution (52:48) as mobile phase, and operating the detection at $30^{\circ} \mathrm{C}$ with flow rate and detection wavelength at $1 \mathrm{~mL} / \mathrm{min}$ and $230 \mathrm{~nm}$, respectively. The HPLC analysis was validated and met the methodological requirements. 
The drug loading $(D L)$ and encapsulation efficiency $(E E)$ of DOC@mPEG-PLA and DOC(Nc)@mPEG-PLA were calculated as below:

$D L=\frac{\text { Amount of DOC loaded }}{\text { Total amout of DOC and excipients loaded }} \times 100 \% \quad E E=\frac{\text { Amount of DOC loaded }}{\text { Amout of DOC used }} \times 100 \%$

\subsubsection{Particle Morphology}

The morphology of docetaxel was performed on a scanning electron microscope (SEM) (Quanta 250, FEI, Hillsboro, OR, USA), after coating the surface of the docetaxel particles with gold. After staining, the samples were fixed on a copper mesh (Ted Pella, Redding, CA, USA) by phosphotungstic acid, the morphology of DOC(Nc), DOC@mPEG-PLA, and DOC(Nc)@mPEG-PLA was observed by using a transmission electron microscope (TEM) (Tecnai Spirit, FEI, Hillsboro, OR, USA).

\subsubsection{Crystalline Study}

The crystalline states of DOC, DOC(Nc), DOC@mPEG-PLA, and DOC(Nc)@mPEGPLA were studied by employing x-ray diffraction (XRD) (Ultima IV, Rigaku, Tokyo, Japan) and differential scanning calorimetry (DSC) (DSC Q2000, TA Instruments, New Castle, $\mathrm{DE}$, America).

\subsection{Release Behaviors In Vitro}

The in vitro release behaviors of DOC, DOC(Nc), DOC@mPEG-PLA, and DOC(Nc)@mPEGPLA were studied by dialysis [35,51]. Firstly, the DOC, DOC(Nc), DOC@mPEG-PLA, and DOC(Nc)@mPEG-PLA were diluted to $100 \mu \mathrm{g} / \mathrm{mL}$ (calculated as docetaxel) with PBS buffer (pH 7.4), and then $2 \mathrm{~mL}$ diluted samples was added into dialysis bags (3000 Da molecular weight cutoff). After immersing these analysis bags into a $30 \mathrm{~mL}$ PBS buffer with stirring speed of $100 \mathrm{rpm}$ at $37^{\circ} \mathrm{C}, 0.5 \mathrm{~mL}$ samples were harvested at $0.5,1,2,4,6,8$, and $12 \mathrm{~h}$. Considering the solubility of docetaxel in water is about $3.9-6.0 \mu \mathrm{g} / \mathrm{mL}$ [52,53], the release medium was replaced by fresh PBS buffer at each pre-determined sampling time point to maintain sink conditions [54]. The DOC concentration in the release buffer was detected via HPLC as exhibited in Section 3.5.2.

\subsection{Stability Evaluation}

The stability of different formulations was studied in this study. Briefly, freshly prepared DOC $(\mathrm{Nc})$, DOC@mPEG-PLA, and DOC(Nc)@mPEG-PLA (without dilution) were performed under room temperature for $0,1,2,3$, and 7 days, and the particle sizes of all samples collected at pre-determined times were analyzed in triplicate. In addition, we evaluated the stability of nanoparticles in a PBS buffer and 10\% $(v / v)$ FBS-PBS buffer after diluting fresh prepared nanoparticles to $100 \mu \mathrm{g} / \mathrm{mL}$ (calculated as docetaxel) and holding at $37^{\circ} \mathrm{C}$ for $8 \mathrm{~h}$ [55].

\subsection{In Vivo Pharmacokinetic Studies}

We employed male Sprague-Dawley (SD) rats to explore the in vivo pharmacokinetic performance of different formulations. Briefly, twenty SD rats, weighing $250 \pm 20 \mathrm{~g}$, were randomly divided into four groups, and intravenously injected with docetaxel injection (prepared similarly to Taxotere ${ }^{\circledR}$; in brief, $80 \mathrm{mg}$ docetaxel was dissolved in $2 \mathrm{~mL}$ polysorbate

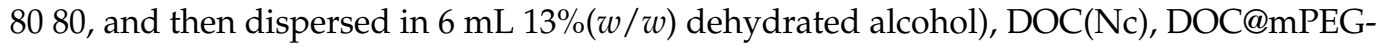
PLA, and DOC(Nc)@mPEG-PLA at $10 \mathrm{mg} / \mathrm{kg}$. After administration for 1, 5, 15, and $30 \mathrm{~min}$; and $1,2,4,6,8,12$, and $24 \mathrm{~h}$, a blood sample of about $0.3 \mathrm{~mL}$ was collected by retro-orbital puncture. The plasma was centrifuged at $4000 \mathrm{rpm}$ for $10 \mathrm{~min}$ at $4{ }^{\circ} \mathrm{C}$ and stored at $-20^{\circ} \mathrm{C}$ until analysis thereafter.

Before pumping the samples into an HPLC-MS system for concentration detection, the plasma samples were treated as follows: $50 \mu \mathrm{L}$ plasma samples, $10 \mu \mathrm{L}$ paclitaxel solution (internal standard, $20 \mu \mathrm{g} / \mathrm{mL}$ ), and $60 \mu \mathrm{L} 0.3 \%$ formic acid-acetonitrile solution were mixed 
by vortexing for $3 \mathrm{~min}$, and then centrifugated at 12,000 rpm for $10 \mathrm{~min}$. The concentration of the supernatant was detected by HPLC-MS/MS performed as below:

Column: Ultimate AQ-C18 column $(2.1 \times 100 \mathrm{~mm}, 1.8 \mu \mathrm{m})$

Mobile phase: $0.1 \%$ formic acid-acetonitrile solution $(\mathrm{A})$ and $0.1 \%$ formic acid-water solution (B)

Gradient sequence: $0.1 \sim 3.0 \mathrm{~min}, 55 \sim 95 \% \mathrm{~B}$; 3.0 3.1 $\mathrm{min}, 95 \sim 55 \% \mathrm{~B}$; $3.1 \sim 6.0 \mathrm{~min}, 55 \% \mathrm{~B}$; at flow rate of $0.3 \mathrm{~mL} / \mathrm{min}$.

MS condition: Turbo ion spray interface voltage, $-4.5 \mathrm{kV}$; turbo heater temperature, $500{ }^{\circ} \mathrm{C}$; atomizing gas pressure, $340.5 \mathrm{kPa}$; auxiliary gas pressure, $345.5 \mathrm{kPa}$; curtain gas pressure, $205.0 \mathrm{kPa}$; decluster voltage, $100 \mathrm{~V}$; collision voltage of docetaxel and paclitaxel, $37 \mathrm{eV}$ and $20 \mathrm{eV} ; \mathrm{m} / \mathrm{z}, 830.37 \rightarrow 549.24$ for docetaxel and $876.36 \rightarrow 286.3$ for paclitaxel.

\subsection{Statistical Analysis}

Pharmacokinetic parameters were calculated using the DAS 3.2.8 pharmacokinetics program (developed by the Clinical Trial Center of Shanghai University of Traditional Chinese Medicine, Shanghai, China). All values are expressed as the means \pm SD. The statistical analysis was performed using one-way ANOVA with SPSS Statistics 22.0 (SPSS Inc., Chicago, IL, USA). The differences were considered significant at $p<0.05$.

\section{Conclusions}

In this study, we constructed docetaxel nanocrystals (DOC(Nc) with a diameter of $168.4 \mathrm{~nm}$ by HPH. We also prepared docetaxel micelles (DOC@mPEG-PLA) and docetaxel nanocrystal-loaded micelles (DOC(Nc)@mPEG-PLA), with particle sizes of $36.3 \mathrm{~nm}$ and $72.5 \mathrm{~nm}$, respectively, by a thin-film hydration method. The results of XRD and DSC showed that the crystallinity of docetaxel decreased after transforming it into nanocrystals, and the docetaxel in DOC@mPEG-PLA and DOC(Nc)@mPEG-PLA were amorphous. The formulations constructed in this paper exhibited good stability in the 7 day stability study period. The bioavailability of docetaxel was enhanced by DOC $(\mathrm{Nc})$ and DOC@mPEG-PLA, while DOC(Nc)@mPEG-PLA had the highest bioavailability, which was 2.69-, 1.39-, and 1.69-fold to DOC injection, DOC $(\mathrm{Nc})$, and DOC@mPEG-PLA, respectively. In addition, compared to DOC injection, DOC (NC), and DOC@mPEG-PLA, DOC(NC)@mPEG-PLA showed decreased CL and enhanced MRT and $t_{1 / 2}$. These results showed that DOC(NC)@mPEGPLA exhibited the optimum pharmacokinetic behavior, and revealed that constructing nanocrystal-loaded micelles is a promising way to enhance the in vivo circulation and bioavailability of rapidly-metabolized drugs like docetaxel.

Author Contributions: M.C. and Q.L. were responsible for investigation and writing the original manuscript; T.G., Y.F. and P.Y. participated in investigation; Y.S. and Y.J. were responsible for data analysis; J.F. participated in supervision; L.T. was responsible for funding acquisition and for the review and editing of the manuscript. All authors have read and agreed to the published version of the manuscript.

Funding: This research was funded by the National Natural Science Foundation of China (81960717), the "1050" Young Talent Scholar discipline project of Jiangxi University of Chinese Medicine (5142001012), and the Ph.D. startup foundation of Jiangxi University of Chinese Medicine (2018BSZR018).

Institutional Review Board Statement: The study was conducted according to the guidelines of the Declaration of Helsinki, and approved by the Institutional Review Board of Jiangxi University of Traditional Chinese Medicine (protocol code: JZLLSC20210020).

Informed Consent Statement: Not applicable.

Data Availability Statement: The data used and/or analyzed during this study are available from the corresponding author upon reasonable request.

Conflicts of Interest: The authors declare no conflict of interest.

Sample Availability: Samples of the formulations are available from the corresponding authors. 


\section{References}

1. Wang, Y.J.; Chen, L.J.; Tan, L.W.; Zhao, Q.; Luo, F.; Wei, Y.Q.; Qian, Z.Y. PEG-PCL based micelle hydrogels as oral docetaxel delivery systems for breast cancer therapy. Biomaterials 2014, 35, 6972-6985. [CrossRef]

2. Prieto-Vila, M.; Shimomura, I.; Kogure, A.; Usuba, W.; Takahashi, R.U.; Ochiya, T.; Yamamoto, Y. Quercetin inhibits Lef1 and resensitizes docetaxel-resistant breast cancer cells. Molecules 2020, 25, 2576. [CrossRef]

3. Zawilska, P.; Machowska, M.; Wisniewski, K.; Grynkiewicz, G.; Hrynyk, R.; Rzepecki, R.; Gubernator, J. Novel pegylated liposomal formulation of docetaxel with 3-n-pentadecylphenol derivative for cancer therapy. Eur. J. Pharm. Sci. 2021, 163, 105838. [CrossRef]

4. Razak, S.A.A.; Gazzali, A.M.; Fisol, F.A.; Abdulbaqi, I.M.; Parumasivam, T.; Mohtar, N.; Wahab, H.A. Advances in nanocarriers for effective delivery of docetaxel in the treatment of lung cancer: An overview. Cancers 2021, 13, 400. [CrossRef]

5. Xu, Y.X.; Fang, T.X.; Yang, Y.F.; Sun, L.A.; Shen, Q. Preparation of deoxycholate-modified docetaxel-cimetidine complex chitosan nanoparticles to improve oral bioavailability. AAPS PharmSciTech 2019, 20, 302. [CrossRef]

6. Ruiz-Gaton, L.; Espuelas, S.; Huarte, J.; Larraneta, E.; Martin-Arbella, N.; Irache, J.M. Nanoparticles from Gantrez ${ }^{\circledR}$ ANpoly(ethylene glycol) conjugates as carriers for oral delivery of docetaxel. Int. J. Pharm. 2019, 571, 118699. [CrossRef]

7. Cui, W.P.; Zhao, H.Q.; Wang, C.; Chen, Y.; Luo, C.; Zhang, S.W.; Sun, B.J.; He, Z.G. Co-encapsulation of docetaxel and cyclosporin A into SNEDDS to promote oral cancer chemotherapy. Drug Deliv. 2019, 26, 542-550. [CrossRef] [PubMed]

8. Liu, H.L.; Tu, L.X.; Zhou, Y.X.; Dang, Z.F.; Wang, L.T.; Du, J.F.; Feng, J.F.; Hu, K.L. Improved bioavailability and antitumor effect of docetaxel by TPGS modified proniosomes: In vitro and in vivo evaluations. Sci. Rep. 2017, 7, 43372. [CrossRef]

9. Guo, X.H.; Zhang, J.Y.; Cai, Q.Q.; Fan, S.T.; Xu, Q.Q.; Zang, J.Y.; Yang, H.T.; Yu, W.J.; Li, Z.; Zhang, Z.Z. Acetic acid transportermediated, oral, multifunctional polymer liposomes for oral delivery of docetaxel. Colloid. Surf. B 2021, 198, 111499. [CrossRef]

10. Tian, C.T.; Guo, J.J.; Wang, G.; Sun, B.J.; Na, K.X.; Zhang, X.B.; Xu, Z.Y.; Cheng, M.S.; He, Z.G.; Sun, J. Efficient intestinal digestion and on site tumor-bioactivation are the two important determinants for chylomicron-mediated lymph-targeting triglyceride-mimetic docetaxel oral prodrugs. Adv. Sci. 2019, 6, 1901810. [CrossRef]

11. Zhu, C.; Gong, S.; Ding, J.; Yu, M.; Ahmad, E.; Feng, Y.; Gan, Y. Supersaturated polymeric micelles for oral silybin delivery: The role of the Soluplus-PVPVA complex. Acta Pharm. Sin. B 2019, 9, 107-117. [CrossRef]

12. Liu, Q.M.; Cheng, M.; Liang, J.Q.; Jin, Y.; Feng, J.F.; Tu, L.X. Enhancing oral bioavailability by paclitaxel polymeric micelles: Role of transmembrane pathways in the oral absorption. J. Biomed. Nanotechnol. 2020, 16, 1160-1168. [CrossRef]

13. Cheng, M.; Liu, Q.M.; Liu, W.; Yuan, F.Y.; Feng, J.F.; Jin, Y.; Tu, L.X. Engineering micelles for the treatment and diagnosis of atherosclerosis. J. Drug Deliv. Sci. Tec. 2021, 63, 102473. [CrossRef]

14. Zheng, X.; Xie, J.Z.; Zhang, X.; Sun, W.T.; Zhao, H.Y.; Li, Y.T.; Wang, C. An overview of polymeric nanomicelles in clinical trials and on the market. Chin. Chem. Lett. 2021, 32, 243-257. [CrossRef]

15. Zoya, I.; He, H.S.; Wang, L.T.; Qi, J.P.; Lu, Y.; Wu, W. The intragastrointestinal fate of paclitaxel-loaded micelles: Implications on oral drug delivery. Chin. Chem. Lett. 2021, 32, 1545-1549. [CrossRef]

16. Zhao, J.; Chai, Y.D.; Zhang, J.; Huang, P.F.; Nakashima, K.; Gong, Y.K. Long circulating micelles of an amphiphilic random copolymer bearing cell outer membrane phosphorylcholine zwitterions. Acta Biomater. 2015, 16, 94-102. [CrossRef]

17. Zheng, P.; Liu, Y.; Chen, J.J.; Xu, W.G.; Li, G.; Ding, J.X. Targeted pH-responsive polyion complex micelle for controlled intracellular drug delivery. Chin. Chem. Lett. 2020, 31, 1178-1182. [CrossRef]

18. Li, W.Q.; Wu, J.Y.; Zhang, J.; Wang, J.J.; Xiang, D.X.; Luo, S.L.; Li, J.H.; Liu, X.Y. Puerarin-loaded PEG-PE micelles with enhanced anti-apoptotic effect and better pharmacokinetic profile. Drug Deliv. 2018, 25, 827-837. [CrossRef]

19. Tam, Y.T.; Shin, D.H.; Chen, K.E.; Kwon, G.S. Poly(ethylene glycol)-block-poly(D, L-lactic acid) micelles containing oligo (lactic acid)(8)-paclitaxel prodrug: In vivo conversion and antitumor efficacy. J. Control. Release 2019, 298, 186-193. [CrossRef]

20. Liang, H.M.; Zou, F.M.; Liu, Q.W.; Wang, B.L.; Fu, L.Y.; Liang, X.F.; Liu, J.; Liu, Q.S. Nanocrystal-loaded liposome for targeted delivery of poorly water-soluble antitumor drugs with high drug loading and stability towards efficient cancer therapy. Int. J. Pharm. 2021, 599, 120418. [CrossRef] [PubMed]

21. Tu, L.X.; Cheng, M.; Sun, Y.B.; Fang, Y.Y.; Liu, J.L.; Liu, W.; Feng, J.F.; Jin, Y. Fabrication of ultra-small nanocrystals by formation of hydrogen bonds: In vitro and in vivo evaluation. Int. J. Pharm. 2020, 573, 118730. [CrossRef]

22. Liu, J.L.; Tu, L.X.; Cheng, M.; Feng, J.F.; Jin, Y. Mechanisms for oral absorption enhancement of drugs by nanocrystals. J. Drug Deliv. Sci. Tec. 2020, 56, 101607. [CrossRef]

23. Lu, Y.; Chen, Y.; Gemeinhart, R.A.; Wu, W.; Li, T. Developing nanocrystals for cancer treatment. Nanomedicine 2015, 10, $2537-2552$. [CrossRef]

24. Mohammad, I.S.; Hu, H.; Yin, L.F.; He, W. Drug nanocrystals: Fabrication methods and promising therapeutic applications. Int. J. Pharm. 2019, 562, 187-202. [CrossRef]

25. Cheng, M.; Yuan, F.Y.; Liu, J.L.; Liu, W.; Feng, J.F.; Jin, Y.; Tu, L.X. Fabrication of fine puerarin nanocrystals by Box-Behnken Design to enhance intestinal absorption. AAPS PharmSciTech 2020, 21, 90. [CrossRef] [PubMed]

26. Ye, Y.H.; Zhang, X.W.; Zhang, T.P.; Wang, H.; Wu, B.J. Design and evaluation of injectable niclosamide nanocrystals prepared by wet media milling technique. Drug Dev. Ind. Pharm. 2015, 41, 1416-1424. [CrossRef] [PubMed]

27. Hollis, C.P.; Weiss, H.L.; Leggas, M.; Evers, B.M.; Gemeinhart, R.A.; Li, T.L. Biodistribution and bioimaging studies of hybrid paclitaxel nanocrystals: Lessons learned of the EPR effect and image-guided drug delivery. J. Control. Release 2013, $172,12-21$. [CrossRef] 
28. Liu, H.Z.; Ma, Y.; Liu, D.; Fallon, J.K.; Liu, F. The Effect of surfactant on paclitaxel nanocrystals: An in vitro and in vivo study. J. Biomed. Nanotechnol. 2016, 12,147-153. [CrossRef]

29. Lu, Y.; Li, Y.; Wu, W. Injected nanocrystals for targeted drug delivery. Acta Pharm. Sin. B 2016, 6, 106-113. [CrossRef] [PubMed]

30. Lu, Y.; Qi, J.P.; Dong, X.C.; Zhao, W.L.; Wu, W. The in vivo fate of nanocrystals. Drug Discov. Today 2017, 22, 744-750. [CrossRef] [PubMed]

31. Kadiu, I.; Nowacek, A.; McMillan, J.; Gendelman, H.E. Macrophage endocytic trafficking of antiretroviral nanoparticles. Nanomedicine 2011, 6, 975-994. [CrossRef]

32. Hao, L.L.; Wang, X.Y.; Zhang, D.R.; Xu, Q.Y.; Song, S.Y.; Wang, F.H.; Li, C.Y.; Guo, H.J.; Liu, Y.; Zheng, D.D.; et al. Studies on the preparation, characterization and pharmacokinetics of Amoitone B Nanocrystals. Int. J. Pharm. 2012, 433, 157-164. [CrossRef]

33. Hao, L.L.; Luan, J.J.; Zhang, D.R.; Li, C.Y.; Guo, H.J.; Qi, L.S.; Liu, X.Q.; Li, T.T.; Zhang, Q. Research on the in vitro anticancer activity and in vivo tissue distribution of Amoitone B nanocrystals. Colloid. Surf. B 2014, 117, 258-266. [CrossRef]

34. Wang, Y.C.; Ma, Y.; Ma, Y.Y.; Du, Y.L.; Liu, Z.P.; Zhang, D.R.; Zhang, Q. Formulation and pharmacokinetics evaluation of puerarin nanocrystals for intravenous delivery. J. Nanosci. Nanotechnol. 2012, 12, 6176-6184. [CrossRef]

35. Wang, T.; Qi, J.P.; Ding, N.; Dong, X.C.; Zhao, W.L.; Lu, Y.; Wang, C.H.; Wu, W. Tracking translocation of self-discriminating curcumin hybrid nanocrystals following intravenous delivery. Int. J. Pharm. 2018, 546, 10-19. [CrossRef]

36. Wang, X.Y.; Guo, Y.L.; Qiu, L.Z.; Wang, X.Y.; Li, T.L.; Han, L.F.; Ouyang, H.Z.; Xu, W.; Chu, K.D. Preparation and evaluation of carboxymethyl chitosan-rhein polymeric micelles with synergistic antitumor effect for oral delivery of paclitaxel. Carbohyd. Polym. 2019, 206, 121-131. [CrossRef]

37. Hou, J.; Sun, E.; Sun, C.Y.; Wang, J.; Yang, L.; Jia, X.B.; Zhang, Z.H. Improved oral bioavailability and anticancer efficacy on breast cancer of paclitaxel via Novel Soluplus ${ }^{\circledR}$-Solutol ${ }^{\circledR}$ HS15 binary mixed micelles system. Int. J. Pharm. 2016, 512, 186-193. [CrossRef]

38. Wang, Y.; Wang, X.X.; Zhang, J.; Wang, L.; Ou, C.Q.; Shu, Y.Q.; Wu, Q.J.; Ma, G.L.; Gong, C.Y. Gambogic acid-encapsulated polymeric micelles improved therapeuticeffects on pancreatic cancer. Chin. Chem. Lett. 2019, 30, 885-888. [CrossRef]

39. Mekseriwattana, W.; Srisuk, S.; Kriangsaksri, R.; Niamsiri, N.; Prapainop, K. The impact of serum proteins and surface chemistry on magnetic nanoparticle colloidal stability and cellular uptake in breast cancer cells. AAPS PharmSciTech 2019, 20, 55. [CrossRef]

40. Hsu, H.J.; Han, Y.X.; Cheong, M.; Kral, P.; Hong, S. Dendritic PEG outer shells enhance serum stability of polymeric micelles. Nanomed-Nanotechnol 2018, 14, 1879-1889. [CrossRef]

41. Wei, L.S.; Ji, Y.X.; Gong, W.; Kang, Z.Q.; Meng, M.; Zheng, A.P.; Zhang, X.Y.; Sun, J.X. Preparation, physical characterization and pharmacokinetic study of paclitaxel nanocrystals. Drug. Dev. Ind. Pharm. 2015, 41, 1343-1352. [CrossRef]

42. Hu, X.; Han, R.; Quan, L.H.; Liu, C.Y.; Liao, Y.H. Stabilization and sustained release of zeylenone, a soft cytotoxic drug, within polymeric micelles for local antitumor drug delivery. Int. J. Pharm. 2013, 450, 331-337. [CrossRef]

43. Jin, Y.; Wu, Z.M.; Li, C.B.; Zhou, W.S.; Shaw, J.P.; Baguley, B.C.; Liu, J.P.; Zhang, W.L. Optimization of weight ratio for DSPEPEG/TPGS hybrid micelles to improve drug retention and tumor penetration. Pharm. Res. 2018, 35, 13. [CrossRef]

44. Hu, Q.Q.; Bai, L.; Zhu, Z.J.; Su, Z.Y.; Bai, P.; Tang, M.H.; Dou, C.X.; Yan, J.F.; Tong, R.S.; Zhang, W.Y.; et al. $\beta$-Elemene-loaded polymeric micelles intensify anti-carcinoma efficacy and alleviate side effects. Chin. Chem. Lett. 2019, 31, 915-918. [CrossRef]

45. Tan, L.W.; Ma, B.Y.; Zhao, Q.; Zhang, L.; Chen, L.J.; Peng, J.R.; Qian, Z.Y. Toxicity evaluation and anti-tumor study of docetaxel loaded mPEG-Polyester micelles for breast cancer therapy. J. Biomed. Nanotechnol. 2017, 13, 393-408. [CrossRef]

46. Guan, Q.X.; Sun, D.D.; Zhang, G.Y.; Sun, C.; Wang, M.; Ji, D.Y.; Yang, W. Docetaxel-loaded self-assembly stearic acid-modified bletilla striata polysaccharide micelles and their anticancer effect: Preparation, characterization, cellular uptake and in vitro evaluation. Molecules 2016, 21, 1641. [CrossRef]

47. Choi, J.S.; Park, J.S. Development of docetaxel nanocrystals surface modified with transferrin for tumor targeting. Drug Des. Dev. Ther. 2017, 11, 17-26. [CrossRef]

48. Lv, F.M.; Wang, J.; Chen, H.N.; Sui, L.; Feng, L.L.; Liu, Z.P.; Liu, Y.; Wei, G.; Lu, W.Y. Enhanced mucosal penetration and efficient inhibition efficacy against cervical cancer of PEGylated docetaxel nanocrystals by TAT modification. J. Control. Release 2021, 336, 572-582. [CrossRef]

49. Wan, X.M.; Beaudoin, J.J.; Vinod, N.; Min, Y.Z.; Makita, N.; Bludau, H.; Jordan, R.; Wang, A.; Sokolsky, M.; Kabanov, A.V. Co-delivery of paclitaxel and cisplatin in poly(2-oxazoline) polymeric micelles: Implications for drug loading, release, pharmacokinetics and outcome of ovarian and breast cancer treatments. Biomaterials 2019, 192, 1-14. [CrossRef]

50. Chen, T.E.; Tu, L.X.; Wang, G.; Qi, N.; Wu, W.; Zhang, W.; Feng, J.F. Multi-functional chitosan polymeric micelles as oral paclitaxel delivery systems for enhanced bioavailability and anti-tumor efficacy. Int. J. Pharm. 2020, 578, 119105. [CrossRef]

51. Chen, M.; Li, W.Q.; Zhang, X.; Dong, Y.; Hua, Y.B.; Zhang, H.; Gao, J.; Zhao, L.; Li, Y.; Zheng, A.P. In vitro and in vivo evaluation of SN-38 nanocrystals with different particle sizes. Int. J. Nanomed. 2017, 12, 5487-5500. [CrossRef]

52. Lim, S.M.; Pang, Z.W.; Tan, H.Y.; Shaikh, M.; Adinarayana, G.; Garg, S. Enhancement of docetaxel solubility using binary and ternary solid dispersion systems. Drug Dev. Ind. Pharm. 2015, 41, 1847-1855. [CrossRef]

53. Hekmat, A.; Attar, H.; Kordi, A.A.S.; Iman, M.; Jaafari, M.R. New oral formulation and in vitro evaluation of docetaxel-loaded nanomicelles. Molecules 2016, 21, 1265. [CrossRef] 
54. Lai, T.C.; Cho, H.; Kwon, G.S. Reversibly core cross-linked polymeric micelleswith pH- and reduction-sensitivities: Effects of cross-linking degree on particle stability, drug release kinetics, and anti-tumor efficacy. Polym. Chem. 2014, 5, 1650-1661. [CrossRef]

55. Hou, X.Y.; Lin, H.; Zhou, X.D.; Cheng, Z.T.; Li, Y.; Liu, X.; Zhao, F.; Zhu, Y.P.; Zhang, P.; Chen, D.Q. Novel dual ROS-sensitive and CD44 receptor targeting nanomicelles based on oligomeric hyaluronic acid for the efficient therapy of atherosclerosis. Carbohyd. Polym. 2020, 232, 115787. [CrossRef] [PubMed] 